International Mathematical Forum, 2, 2007, no. 61, 3021 - 3026

\title{
A Construction of Some Symmetric Designs with Parameters $(196,91,42)$
}

\author{
Dean Crnković \\ Department of Mathematics \\ Faculty of Philosophy, University of Rijeka \\ Omladinska 14, 51000 Rijeka, Croatia \\ deanc@ffri.hr
}

\begin{abstract}
Let $q$ be a prime power, $q \equiv 1(\bmod 4)$, and let $p=\frac{q+1}{2}$. We construct an orbit structure for the parameters $\left(4 p^{2}, 2 p^{2}-p, p^{2}-p\right)$ and the orbit size distribution with $q+1$ fixed points and $2 q$ orbits of size $p$. Further, we describe a construction of eight symmetric designs with parameters $(196,91,42)$ admitting an automorphism group isomorphic to Frob $_{7.3} \times Z_{6}$.
\end{abstract}

Mathematics Subject Classification: 05B05

Keywords: symmetric design, Menon design, Hadamard matrix

\section{Introduction}

A symmetric $(v, k, \lambda)$ design is a finite incidence structure $(\mathcal{P}, \mathcal{B}, I)$, where $\mathcal{P}$ and $\mathcal{B}$ are disjoint sets and $I \subseteq \mathcal{P} \times \mathcal{B}$, with the following properties:

1. $|\mathcal{P}|=|\mathcal{B}|=v$;

2. every element of $\mathcal{B}$ is incident with exactly $k$ elements of $\mathcal{P}$;

3. every pair of distinct elements of $\mathcal{P}$ is incident with exactly $\lambda$ elements of $\mathcal{B}$.

The elements of the set $\mathcal{P}$ are called points and the elements of the set $\mathcal{B}$ are called blocks.

Let $\mathcal{D}=(\mathcal{P}, \mathcal{B}, I)$ be a symmetric $(v, k, \lambda)$ design and $G$ a subgroup of $A u t \mathcal{D}$. The action of $G$ produces the same number of point and block orbits (see [7, Theorem 3.3, p. 79]). We denote that number by $t$, the point orbits 
by $\mathcal{P}_{1}, \ldots, \mathcal{P}_{t}$, the block orbits by $\mathcal{B}_{1}, \ldots, \mathcal{B}_{t}$, and put $\left|\mathcal{P}_{r}\right|=\omega_{r}$ and $\left|\mathcal{B}_{i}\right|=$ $\Omega_{i}$. We shall denote the points of the orbit $\mathcal{P}_{r}$ by $r_{0}, \ldots, r_{\omega_{r}-1}$, (i.e. $\mathcal{P}_{r}=$ $\left.\left\{r_{0}, \ldots, r_{\omega_{r}-1}\right\}\right)$. Further, we denote by $\gamma_{i r}$ the number of points of $\mathcal{P}_{r}$ which are incident with a representative of the block orbit $\mathcal{B}_{i}$. The numbers $\gamma_{i r}$ are independent of the choice of the representative of the block orbit $\mathcal{B}_{i}$. For those numbers the following equalities hold (see [6]):

$$
\begin{gathered}
\sum_{r=1}^{t} \gamma_{i r}=k \\
\sum_{r=1}^{t} \frac{\Omega_{j}}{\omega_{r}} \gamma_{i r} \gamma_{j r}=\lambda \Omega_{j}+\delta_{i j} \cdot(k-\lambda) .
\end{gathered}
$$

Definition 1.1 Let $(\mathcal{D})$ be a symmetric $(v, k, \lambda)$ design and $G \leq$ Aut $\mathcal{D}$. Further, let $\mathcal{P}_{1}, \ldots, \mathcal{P}_{t}$ be the point orbits and $\mathcal{B}_{1}, \ldots, \mathcal{B}_{t}$ the block orbits with respect to $G$, and let $\omega_{1}, \ldots, \omega_{t}$ and $\Omega_{1}, \ldots, \Omega_{t}$ be the respective orbit lengths. We call $\left(\mathcal{P}_{1}, \ldots, \mathcal{P}_{t}\right)$ and $\left(\mathcal{B}_{1}, \ldots, \mathcal{B}_{t}\right)$ the orbit distributions, and $\left(\omega_{1}, \ldots, \omega_{t}\right)$ and $\left(\Omega_{1}, \ldots, \Omega_{t}\right)$ the orbit size distributions for the design and the group $G$. A $(t \times t)$-matrix $\left(\gamma_{i r}\right)$ with entries satisfying conditions $(1)$ and $(2)$ is called an orbit structure for the parameters $(v, k, \lambda)$ and orbit distributions $\left(\mathcal{P}_{1}, \ldots, \mathcal{P}_{t}\right)$ and $\left(\mathcal{B}_{1}, \ldots, \mathcal{B}_{t}\right)$.

The first step - when constructing designs for given parameters and orbit distributions - is to find all compatible orbit structures $\left(\gamma_{i r}\right)$. The next step, called indexing, consists in determining exactly which points from the point orbit $\mathcal{P}_{r}$ are incident with a chosen representative of the block orbit $\mathcal{B}_{i}$ for each number $\gamma_{i r}$. Because of the large number of possibilities, it is often necessary to involve a computer in both steps of the construction.

Definition 1.2 The set of all indices of points of the orbit $\mathcal{P}_{r}$ which are incident with a fixed representative of the block orbit $\mathcal{B}_{i}$ is called the index set for the position $(i, r)$ of the orbit structure and the given representative.

A Hadamard matrix of order $m$ is an $(m \times m)$ matrix $H=\left(h_{i, j}\right), h_{i, j} \in$ $\{-1,1\}$, satisfying $H H^{T}=H^{T} H=m I_{m}$, where $I_{m}$ is an $(m \times m)$ identity matrix. A Hadamard matrix is regular if the row and column sums are constant. It is well known that the existence of a symmetric design with parameters $\left(4 u^{2}, 2 u^{2}-u, u^{2}-u\right)$ is equivalent to the existence of a regular Hadamard matrix of order $4 u^{2}$ (see [10, Theorem 1.4 pp. 280]). Such symmetric designs are called Menon designs. 


\section{Paley Partial Difference Sets and Orbit Struc- tures}

Let $q$ be a prime power, $q \equiv 1(\bmod 4)$, and $C=\left(c_{i j}\right)$ be a $(q \times q)$ matrix defined as follows:

$$
c_{i j}= \begin{cases}1, & \text { if }(i-j) \text { is a nonzero square in } F_{q}, \\ 0, & \text { otherwise. }\end{cases}
$$

$C$ is a symmetric matrix, since -1 is a square in $F_{q}$. There are as many nonzero squares as nonsquares in $F_{q}$, so each row of $C$ has $\frac{q-1}{2}$ elements equal 1 and $\frac{q+1}{2}$ zeros. The set of nonzero squares in $F_{q}$ is a partial difference set, called a Paley partial difference set (see [1, 10.15 Example, pp. 231]). Let $C_{i}$ and $C_{j}$, $i \neq j$, be the $i^{t h}$ and the $j^{\text {th }}$ row of the matrix $C$, respectively. Then

$$
C_{i} \cdot C_{j}^{T}= \begin{cases}\frac{q-1}{4}, & \text { if } c_{i j}=c_{j i}=0, \\ \frac{q-1}{4}-1, & \text { if } c_{i j}=c_{j i}=1 .\end{cases}
$$

The matrix $\bar{C}-I_{q}$ has the same property, where $\bar{C}=\left(\bar{c}_{i j}\right)$ is a $(q \times q)$ matrix such that $\bar{c}_{i j}=c_{i j}+1(\bmod 2)$. Let $\bar{C}_{i}$ and $\bar{C}_{j}, i \neq j$, be the $i^{\text {th }}$ and the $j^{\text {th }}$ row of the matrix $\bar{C}$, respectively. Then

$$
\bar{C}_{i} \cdot \bar{C}_{j}^{T}= \begin{cases}\frac{q-1}{4}, & \text { if } \bar{c}_{i j}=\bar{c}_{j i}=0 \\ \frac{q-1}{4}+1, & \text { if } \bar{c}_{i j}=\bar{c}_{j i}=1 .\end{cases}
$$

The matrix $C+I_{q}$ has the same property.

Further, for $i^{\text {th }}$ row of the matrix $C$ and the $j^{\text {th }}$ row of the matrix $\bar{C}$ the following property holds:

$$
C_{i} \cdot \bar{C}_{j}^{T}= \begin{cases}0, & \text { if } i=j, \\ \frac{q-1}{4}, & \text { if } c_{i j}=c_{j i}=0, i \neq j, \\ \frac{q-1}{4}+1, & \text { if } c_{i j}=c_{j i}=1 .\end{cases}
$$

Lemma 2.1 Let $q$ be a prime power, $q \equiv 1(\bmod 4)$, and let the matrices $C$ and $\bar{C}$ be defined as above. Then the following properties hold:

$$
\begin{gathered}
C \cdot\left(C+I_{q}\right)^{T}=\bar{C} \cdot\left(\bar{C}-I_{q}\right)^{T}=\frac{q-1}{4} J_{q}+\frac{q-1}{4} I_{q}, \\
C \cdot\left(\bar{C}-I_{q}\right)^{T}=\frac{q-1}{4} J_{q}-\frac{q-1}{4} I_{q},
\end{gathered}
$$

where $J_{q}$ is the all-one matrix of dimension $(q \times q)$.

Proof The equalities follow from the properties of the matrices $C, C+I_{q}$, $\bar{C}$, and $\bar{C}-I_{q}$ listed above.

For a positive integer $n$ we denote by $j_{n}$ the all-one vector of dimension $n$, by $0_{n}$ the zero-vector of dimension $n$, and by $0_{n \times n}$ the zero-matrix of dimension $n \times n$. 
Lemma 2.2 Let $q$ be a prime power, $q \equiv 1(\bmod 4)$, and let $p=\frac{q+1}{2}$. Then the matrices

$$
\begin{gathered}
O S_{1}=\left[\begin{array}{c|c|c|c}
0 & 0_{q}^{T} & p j_{q}^{T} & 0_{q}^{T} \\
\hline 0_{q} & 0_{q \times q} & p\left(\bar{C}-I_{q}\right) & p \bar{C} \\
\hline j_{q} & C & \frac{p-1}{2} J_{q}+\frac{p-1}{2} I_{q} & \frac{p-1}{2} C+\frac{p+1}{2}\left(\bar{C}-I_{q}\right) \\
\hline 0_{q} & C+I_{q} & \frac{p+1}{2} C+\frac{p-1}{2}\left(\bar{C}-I_{q}\right) & \frac{p-1}{2} J_{q}+\frac{p-1}{2} I_{q}
\end{array}\right] \\
O S_{2}=\left[\begin{array}{c|c|c|c}
0 & 0_{q}^{T} & p j_{q}^{T} & 0_{q}^{T} \\
\hline 0_{q} & 0_{q \times q} & p\left(\bar{C}-I_{q}\right) & p \bar{C} \\
\hline 0_{q} & \bar{C} & \frac{p-1}{2} J_{q}+\frac{p-1}{2} I_{q} & \frac{p-1}{2} C+\frac{p+1}{2}\left(\bar{C}-I_{q}\right) \\
\hline j_{q} & \bar{C}-I_{q} & \frac{p+1}{2} C+\frac{p-1}{2}\left(\bar{C}-I_{q}\right) & \frac{p-1}{2} J_{q}+\frac{p-1}{2} I_{q}
\end{array}\right]
\end{gathered}
$$

are orbit structures for the parameters $\left(4 p^{2}, 2 p^{2}-p, p^{2}-p\right)$ and the orbit size distribution with $q+1$ fixed points and $2 q$ orbits of size $p$ for points and blocks.

Proof From Lemma 1 and the other properties of the matrices $C, C+I_{q}$, $\bar{C}$, and $\bar{C}-I_{q}$ listed above follows that the matrices $O S_{1}$ and $O S_{2}$ satisfy the equalities (1) and (2).

\section{Symmetric $(196,91,42)$ Designs}

It is known that Menon designs with parameters $\left(4 n^{2}, 2 n^{2}-n, n^{2}-n\right)$ exist whenever $2 n-1$ and $2 n+1$ are both prime powers (see [9]). Symmetric $(196,91,42)$ designs are the smallest Menon designs that do not belong to that familly of Menon designs, since 15 is not a prime power. A.E. Brower and J.H. van Lint had constructed the first symmetric $(196,91,42)$ design on 1983 (see [9]). Further symmetric designs with parameters $(196,91,42)$ are described in [3]. As far as we know these are the only known symmetric $(196,91,42)$ designs.

In our construction of symmetric $(196,91,42)$ designs we use the group

$$
G=\left\langle\rho, \sigma, \tau \mid \rho^{7}=1, \sigma^{3}=1, \tau^{6}=1, \rho^{\sigma}=\rho^{2}, \rho^{\tau}=\rho, \sigma^{\tau}=\sigma\right\rangle
$$

isomorphic to Frob $_{7.3} \times Z_{6}$. We shall assume that an automorphism group $H \leq G$ isomorphic to Frob $_{7.3}$ acts on the symmetric $(196,91,42)$ designs to be constructed with 14 fixed points (and blocks), and 26 orbits of size 7 . That means that the permutation of order 3 has precisely 40 fixed points and 40 fixed blocks. Orbit structures of the type $O S_{1}$ and $O S_{2}$, for $p=7$ and $q=13$, corresponds to such action of $H$ on a symmetric $(196,91,42)$ design. We shall proceed with indexing of the orbit structures $O S_{1}$ and $O S_{2}$ by the method described in [5], having in mind the action of $\tau$ on the $H$-orbits, as described in $[2]$.

Denote the points by $1_{0}, \ldots, 14_{0}, 15_{i} \ldots, 40_{i}, i=0,1,2,3,4,5,6$, and put $G$ $=\langle\rho, \sigma, \tau\rangle$, where the generators for $G$ are permutations defined as follows: 


$$
\begin{aligned}
\rho= & \left(I_{0}\right)\left(K_{0} K_{1} K_{2} K_{3} K_{4} K_{5} K_{6}\right), I=1, \ldots, 14, K=15, \ldots 40, \\
\sigma= & \left(I_{0}\right)\left(K_{0}\right)\left(K_{1} K_{2} K_{4}\right)\left(K_{3} K_{6} K_{5}\right), I=1, \ldots, 14, K=15, \ldots 40, \\
\tau= & \left(1_{0}\right)\left(2_{0}\right)\left(3_{0} 6_{0} 5_{0} 14_{0} 11_{0} 12_{0}\right)\left(4_{0} 10_{0} 8_{0} 13_{0} 7_{0} 9_{0}\right) \\
& \left(15_{i}\right)\left(16_{i} 19_{i} 18_{i} 27_{i} 24_{i} 25_{i}\right)\left(17_{i} 23_{i} 21_{i} 26_{i} 20_{i} 22_{i}\right) \\
& \left(28_{i}\right)\left(29_{i} 32_{i} 31_{i} 40_{i} 37_{i} 38_{i}\right)\left(30_{i} 36_{i} 34_{i} 39_{i} 33_{i} 35_{i}\right), i=0,1,2,3,4,5,6 .
\end{aligned}
$$

As representatives for the block orbits we chose blocks fixed by $\langle\sigma\rangle$. Therefore, the index sets which could occur in the designs are among the following: $0=\emptyset, \quad 1=\{1,2,4\}, \quad 2=\{3,5,6\}, \quad 3=\{0,1,2,4\}, \quad 4=\{0,3,5,6\}$ $5=\{1,2,3,4,5,6\}$.

The indexing process of the orbit structure $O S_{1}$ leads to four mutually non-isomorphic designs, denoted by $\mathcal{D}_{1}, \mathcal{D}_{2}, \mathcal{D}_{3}$, and $\mathcal{D}_{4}$. The designs $\mathcal{D}_{i}$, $i=1,2,3,4$, are self-dual. Orbit structure $O S_{2}$ also produces four mutually non-isomorphic designs. Let us denote these designs by $\mathcal{D}_{5}, \mathcal{D}_{6}, \mathcal{D}_{7}$, and $\mathcal{D}_{8}$. The designs $\mathcal{D}_{7}$ and $\mathcal{D}_{8}$ are dual designs of $\mathcal{D}_{5}$ and $\mathcal{D}_{6}$, respectively.

The designs $\mathcal{D}_{1}, \mathcal{D}_{3}, \mathcal{D}_{5}$, and $\mathcal{D}_{7}$ have the full automorphism group of order 1638, isomorphic to $\mathrm{Frob}_{7.3} \times \mathrm{Frob}_{13.6}$. These designs are isomorphic to the four designs admitting the full automorphism group isomorphic to $F o b_{7 \cdot 3} \times F r_{13.6}$ described in [3]. The designs $\mathcal{D}_{2}, \mathcal{D}_{4}, \mathcal{D}_{6}$, and $\mathcal{D}_{8}$ have the full automorphism group of order 126, isomorphic to $F_{r o b} \cdot 3 \times Z_{6}$. A computer program by Vladimir D. Tonchev [8] computes the order as well as generators of the full automorphism group for each of the designs found. The group structures have been determined with the help of GAP [4].

We shall write down base blocks for the designs $\mathcal{D}_{2}, \mathcal{D}_{4}$, and $\mathcal{D}_{6}$ in terms of the index sets defined above. Since the indexing the fixed part of an orbit stucture is a trivial task, we write down base blocks omitting the fixed part. It is sufficient to write down representatives of the $15^{\text {th }}, 16^{\text {th }}, 17^{\text {th }}, 28^{\text {th }}, 29^{\text {th }}$, and the $30^{t h} H$-orbit, since the other $H$-orbits could be obtained as their $\langle\tau\rangle$ images.

$\mathcal{D}_{2}$ 51211222211210141144441141 15121122221121024113344124 22511112212124101411444411 03233222233235212211112212 30423311223422521221111221 23032332222331152222112121
$\mathcal{D}_{4}$ 51211222211210232233332232 15221111221222023223333223 21512112222113102422334321 04144111144145212211112212 40414411114412511222211211 13042441121431252122111122 


$\mathcal{D}_{6}$
51211222211210141144441141
15121122221121024113344124
22511112212124101411444411
03233222233235212211112212
30423311223422521221111221
23032332222331152222112121

\section{References}

[1] T. Beth, D. Jungnickel and H. Lenz, Design Theory, Cambridge University Press, Cambridge, 1999.

[2] D. Crnković, Symmetric $(70,24,8)$ designs having $F r o b_{21} \times Z_{2}$ as an automorphism group, Glasnik Matematički, 34 (54) (1999), 39-?45.

[3] D. Crnković, Some New Menon Designs with Parameters $(196,91,42)$, Mathematical Communications, 10 (2005), 169-175.

[4] The GAP Group, GAP - Groups, Algorithms, and Programming, Version 4.4.9; 2006. (http://www.gap-system.org)

[5] Z. Janko, Coset Enumeration in Groups and Constructions of Symmetric Designs, Combinatorics '90 (1992), 275-277.

[6] Z. Janko and Tran van Trung, Construction of a new symmetric block design for $(78,22,6)$ with the help of tactical decomposition, Journal of Combinatorial Theory, Series A, 40 (1985), 451-455.

[7] E. Lander, Symmetric Designs: An Algebraic Approach, Cambridge University Press, Cambridge, 1983.

[8] V.D. Tonchev, private communication via Z. Janko.

[9] Tran van Trung, Symmmetric Designs, in: Handbook of Combinatorial Designs, $2^{\text {nd }}$ ed., (C.J. Colbourn and J.H. Dinitz, Eds.), Chapman \& Hall/CRC, 2007, 75-87.

[10] W.D. Wallis, A.P. Street and J.S. Wallis, Combinatorics: Room Squares, Sum-Free Sets, Hadamard matrices, Springer Verlag, Berlin-HeidelbergNew York, 1972.

\section{Received: June 5, 2007}

